\title{
Searches for charged Higgs bosons in CMS; Run-1 legacy and Run-2 results
}

\author{
Alexandros Attikis* on behalf of the CMS Collaboration \\ University of Cyprus \\ E-mail: Alexandros.Attikisecern.ch
}

\begin{abstract}
An overview of the results from various searches for charged Higgs bosons $\left(\mathrm{H}^{ \pm}\right)$is presented, based on data from proton-proton collisions delivered by the CERN LHC and recorded by the CMS experiment. These include the first running period (Run 1) legacy results at a center-of-mass energy $\sqrt{s}=8 \mathrm{TeV}$ and corresponding to an integrated luminosity of $19.7 \mathrm{fb}^{-1}$, as well as the most recent results with the second running period (Run 2) at $\sqrt{s}=13 \mathrm{TeV}$ and corresponding to an integrated luminosity of $35.9 \mathrm{fb}^{-1}$. Model-independent upper limits at $95 \%$ confidence level on the branching ratio and/or the production cross section times the branching ratio for various processes are summarised, for charged Higgs bosons in the mass range of $80 \mathrm{GeV}$ up to $3 \mathrm{TeV}$. For the first time in CMS, the intermediate-mass range near the top quark mass is also explored. A summary of the interpretation of these exclusion limits in the context of the minimal supersymmetric standard model (MSSM) for specific benchmark scenarios is also presented.
\end{abstract}

Prospects for Charged Higgs Discovery at Colliders - CHARGED2018

25-28 September 2018

Uppsala, Sweden

*Speaker. 


\section{Introduction}

In 2012 the ATLAS and CMS experiments delivered the breakthrough discovery of the Higgs boson with a mass of $125 \mathrm{GeV}$ [1 -3], and provided strong evidence for the spontaneous symmetry breaking via the Brout-Englert-Higgs (BEH) mechanism [4-9]. To date, all measurements related to this particle's properties such as mass, coupling, $\mathrm{CP}$ quantum numbers, and production rates do not indicate deviations from the standard model (SM) Higgs boson hypothesis [10 - 14]. Nevertheless, more high-precision measurements are required to reveal, or exclude, differences with respect to the SM predictions, and it is likely that these will only be possible with the high luminosity upgrade of the Large Hadron Collider (LHC), known as the High-Luminosity LHC (HL-LHC).

Many extensions of the SM predict a more complex Higgs sector with several Higgs fields, resulting in a spectrum of Higgs bosons with different masses, charges, and other properties. The existence of charged Higgs boson(s) is inevitable in models with two (or more) Higgs doublets and thus their discovery would constitute unambiguous evidence for new physics beyond the standard model (BSM). In two-Higgs-doublet Models (2HDMs) five physical Higgs bosons are predicted of which three are electrically neutral $\left(\mathrm{h}^{0}, \mathrm{H}^{0}\right.$, and $\left.\mathrm{A}^{0}\right)$ and two are electrically charged $\left(\mathrm{H}^{+}\right.$and $\left.\mathrm{H}^{-}\right)$. Four scenarios are encompassed, which depend on the Higgs boson couplings to fermions. In the type I scenario, all quarks and leptons couple only to the second doublet, while in a type II scenario the second doublet couples to up-type quarks all down-type quarks and charged leptons couple to the first doublet. In the type $\mathrm{X}$ (or "lepton-specific") scenario, both up- and down-type quarks couple to the second doublet and all leptons to the first one. Finally, in the type Y (or "flipped") scenario the roles of the two doublets are reversed with respect to type II. As shown in Fig. 1, in charged Higgs boson decays with $m_{\mathrm{H}^{ \pm}}=150 \mathrm{GeV}$ the $\mathrm{H}^{+} \rightarrow \tau^{+} \bar{v}_{\tau}$ decay is dominant in the type I, type II, and type $\mathrm{X}$ scenarios for $\tan \beta \gtrsim 1$, while hadronic decay modes can also be dominant in the type Y scenarios [15]. The minimal supersymmetric standard model (MSSM) is a special case of a type II scenario [16]. Charged Higgs bosons are also predicted by more complex models, such as triplet models [17-19].

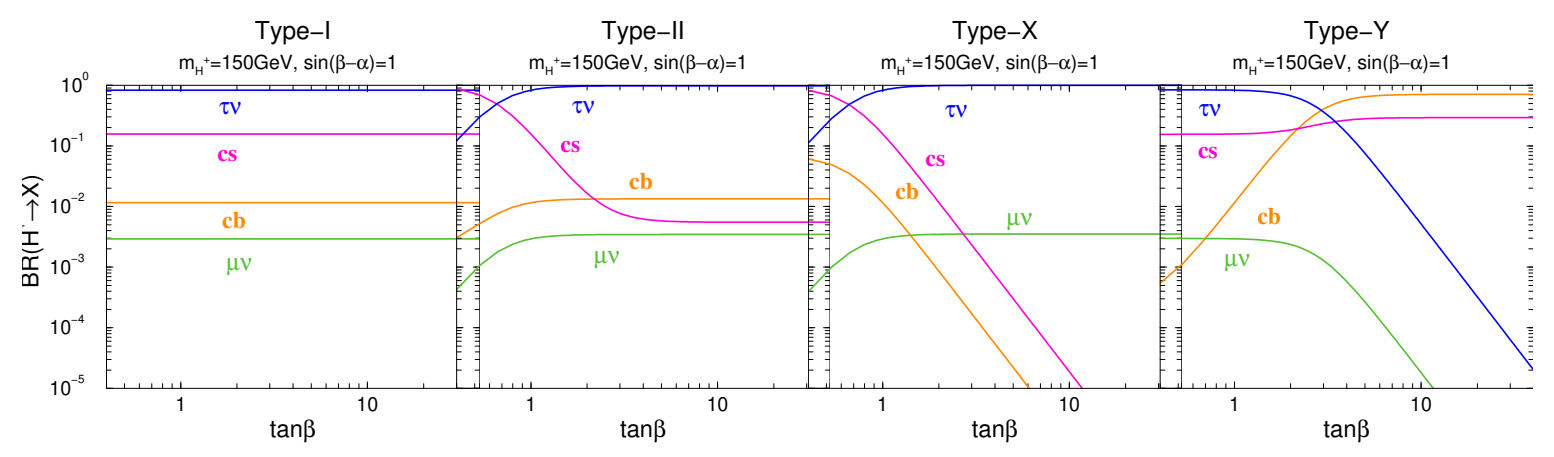

Figure 1: Decay branching ratios of $\mathrm{H}^{ \pm}$in the four different types of $2 \mathrm{HDMs}$, as a function of $\tan \beta$ and for $m_{\mathrm{H}^{ \pm}}=150 \mathrm{GeV}$ and $M=149 \mathrm{GeV}$. The SM-like limit $\sin (\beta-\alpha)=1$ is taken [20].

The dominant $\mathrm{H}^{ \pm}$production mechanism depends on its mass, which is classified into three distinct regions; light, intermediate, and heavy. The light-mass region refers to charged Higgs Higgs bosons with a mass less than that of the top quark $\left(m_{\mathrm{H}^{+}}<m_{\mathrm{t}}-m_{\mathrm{b}}\right)$, which are predominantly 
produced in double-resonant top quark production. In the heavy-mass region $\left(m_{\mathrm{H}^{+}}>m_{\mathrm{t}}-m_{\mathrm{b}}\right)$ the charged Higgs bosons are produced in single-resonant top quark production. In the intermediatemass region $\left(m_{\mathrm{H}^{+}} \sim m_{\mathrm{t}}\right)$, the aforementioned resonant top quark production modes interfere with non-resonant processes, as well as neutral Higgs boson diagrams [21]. Examples of leading order (LO) diagrams describing the $\mathrm{H}^{ \pm}$production at the LHC for the different mass regions are shown in Fig. 2. In these figures, as well as throughout the text, charge conjugated states are implied.

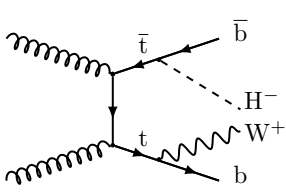

(a)

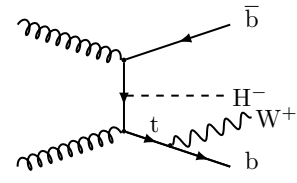

(b)

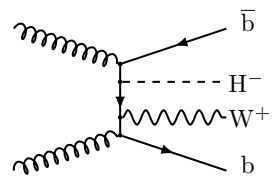

(c)

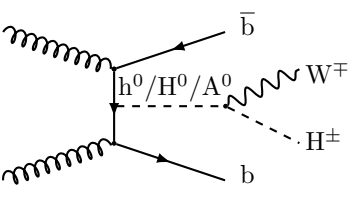

(d)

Figure 2: LO Feynman diagrams describing charged Higgs boson production at the LHC. For light $\mathrm{H}^{ \pm}$and heavy $\mathrm{H}^{ \pm}$, the single-resonant (a) and double-resonant (b) top quark production is dominant, respectively. For intermediate $\mathrm{H}^{ \pm}$, the additional contribution from non-resonant top quark (c) and neutral Higgs boson (d) productions must also be taken into account.

\section{The CMS detector and event reconstruction}

The central feature of the CMS apparatus is a superconducting solenoid of $6 \mathrm{~m}$ internal diameter, providing a magnetic field of $3.8 \mathrm{~T}$. Within the solenoid volume are a silicon pixel and strip tracker, a lead tungstate crystal electromagnetic calorimeter (ECAL) and a brass and scintillator hadronic calorimeter (HCAL), each composed of a barrel and two endcap sections. Forward calorimeters extend the pseudorapidity $(\eta)$ coverage provided by the barrel and endcap detectors up to $|\eta|=5$. Muons are measured in gas-ionisation chambers embedded in the steel flux-return yoke outside the solenoid. A more detailed description of the CMS detector, together with a definition of the coordinate system used and the relevant kinematic variables, can be found in Ref. [22].

Event reconstruction is based on the particle-flow (PF) algorithm [23], which takes full advantage of the CMS apparatus features that include a highly-segmented tracker, a fine-grained ECAL, a hermetic HCAL, a strong magnetic field, and an excellent muon spectrometer. For each collision, the PF algorithm delivers a comprehensive list of identified and reconstructed final-state particles classified into muons, electrons, photons, and charged and neutral hadrons, as well as higher-level objects such as jets, hadronic taus $\left(\tau_{\mathrm{h}}\right)$, and the missing transverse momentum vector $\vec{p}_{\mathrm{T}}^{\text {miss }}$.

The anti- $k_{\mathrm{T}}$ algorithm $[24,25]$ with a distance parameter of $\mathrm{R}=0.4(\mathrm{R}=0.5)$ at $8 \mathrm{TeV}(13 \mathrm{TeV})$ is used in clustering PF candidates into jets. These are used as seeds to identify hadronically decaying tau leptons, which are reconstructed with the hadrons-plus-strips (HPS) algorithm [26, 27]. Jets consistent with originating from a heavy-flavour hadron are identified using the combined secondary vertex (CSV) b tagging algorithm [28]. The scalar transverse momentum $p_{\mathrm{T}}$ sum of all selected jets in an event is denoted as $H_{\mathrm{T}}$, while $\vec{p}_{\mathrm{T}}^{\text {miss }}$ is defined as the projection onto the plane perpendicular to the beam axis of the negative vector sum of the momenta of all reconstructed PF candidates [29]. Its magnitude is referred to as $p_{\mathrm{T}}^{\text {miss }}$. 


\section{Run 1 legacy results}

The discovery of the Higgs boson has been an important success of the LHC first running period (Run 1), but many important searches for charged Higgs bosons were also performed and have set stringent model-independent and model-dependent upper limits for various models. The first ever search for light charged Higgs bosons originating from the decay of a top quark $\left(\mathrm{t} \rightarrow \mathrm{bH}^{+}\right)$ which subsequently decays to a charm and a bottom quark $\left(\mathrm{H}^{+} \rightarrow \mathrm{c} \bar{b}\right)$ was performed using data recorded by CMS with an integrated luminosity of $19.7 \mathrm{fb}^{-1}$ and at center-of-mass energy $\sqrt{s}=8$ $\mathrm{TeV}$. The search of this decay mode, which is enhanced in the type Y scenario of 2HDMs, was based on the selection of $\bar{t}$ events in a final state containing at least four jets of which at least two were identified as originating from the hadronisation of a b quark (b-tagged), a charged lepton $(\ell=$ $\mathrm{e}, \mu)$, and $p_{\mathrm{T}}^{\text {miss }}[30]$. A kinematic fit was performed to identify the dijet combination least likely to be the $b$ quarks originating from direct top quark decays. The invariant mass of this dijet was used as the final observable in a binned maximum likelihood (ML) fit, performed simultaneously to all the observed dijet mass distributions, using the signal and background templates extracted from simulation or data. Combining both channels yielded upper limits at $95 \%$ confidence level (CL) on the branching ratio $\mathscr{B}\left(\mathrm{t} \rightarrow \mathrm{bH}^{+}\right)$of $0.8-0.5 \%$ for $m_{\mathrm{H}^{ \pm}}=90-150 \mathrm{GeV}$ as shown in Fig. 3 (left), assuming that $\mathscr{B}\left(\mathrm{H}^{+} \rightarrow \mathrm{c} \overline{\mathrm{b}}\right)=100 \%$ and $\mathscr{B}\left(\mathrm{t} \rightarrow \mathrm{bH}^{+}\right)+\mathscr{B}\left(\overline{\mathrm{t}} \rightarrow \overline{\mathrm{b}} \mathrm{W}^{-}\right)=100 \%$.

The same data sample was also used to perform a search for a light charged Higgs boson produced in the top quark decays $\left(\mathrm{t} \rightarrow \mathrm{bH}^{+}\right)$and subsequently decaying into a charm quark and a strange antiquark $\left(\mathrm{H}^{+} \rightarrow \mathrm{c} \overline{\mathrm{s}}\right)$. In $2 \mathrm{HDMs}$ of type $\mathrm{I}$ and type $\mathrm{Y}, \mathscr{B}\left(\mathrm{H}^{+} \rightarrow \mathrm{c} \overline{\mathrm{s}}\right)$ is larger than $10 \%$ for any value of $\tan \beta$, while in type II and type $\mathrm{X}$ it can reach $100 \%$ for $\tan \beta<1$. In this search, $\mathrm{t} \overline{\mathrm{t}}$ events were selected in a final state containing a charged lepton $(\ell=\mathrm{e}, \mu)$, at least four jets of which at least two were b-tagged, and $p_{\mathrm{T}}^{\text {miss }}$ [31]. The strategy was to reconstruct the $\mathrm{H}^{+}\left(\mathrm{W}^{+}\right)$in the case of signal (in the case of SM t⿱t) ), with the other $\mathrm{W}$ boson decaying into a lepton-neutrino pair. To fully reconstruct the selected events a kinematic fit was performed, constraining the event to the hypothesis for the production of two top quarks and resulting in an improved mass resolution of the hadronically decaying boson. A binned ML fit to the dijet mass distributions was performed in order to search for a possible signal. The $95 \% \mathrm{CL}$ upper limits on $\mathscr{B}\left(\mathrm{t} \rightarrow \mathrm{bH}^{+}\right)$were set to $1.2-6.5 \%$ for $m_{\mathrm{H}^{ \pm}}=90-160 \mathrm{GeV}$ as shown in Fig. 3 (right), assuming $\mathscr{B}\left(\mathrm{H}^{+} \rightarrow \mathrm{c} \overline{\mathrm{s}}\right)=100 \%$.

The charged Higgs boson production in $\mathrm{t} \overline{\mathrm{t}}$ decays was also studied assuming the $\mathrm{H}^{+} \rightarrow \tau^{+} \bar{v}_{\tau}$ and $\mathrm{H}^{+} \rightarrow \mathrm{t} \overline{\mathrm{b}}$ decay modes, using the $\tau_{\mathrm{h}}+$ jets, $\mu \tau_{\mathrm{h}}, \ell+$ jets, and $\ell \ell^{\prime}$ final states [32]. Data recorded at $\sqrt{s}=8 \mathrm{TeV}$ and corresponding to an integrated luminosity of $19.7 \mathrm{fb}^{-1}$ were analysed and found to agree with the SM expectations. Model-independent limits were derived for the $\mathrm{H}^{+} \rightarrow$ $\tau^{+} \bar{v}_{\tau}$ decay mode in the $\tau_{\mathrm{h}}+$ jets final state, without an assumption on the charged Higgs boson branching ratios. The $95 \% \mathrm{CL}$ upper limits of $\mathscr{B}\left(\mathrm{t} \rightarrow \mathrm{bH}^{+}\right) \cdot \mathscr{B}\left(\mathrm{H}^{+} \rightarrow \tau^{+} \bar{v}_{\tau}\right)=1.2-0.15 \%$ and $\sigma\left(\mathrm{pp} \rightarrow \overline{\mathrm{t}}(\mathrm{b}) \mathrm{H}^{+}\right) \cdot \mathscr{B}\left(\mathrm{H}^{+} \rightarrow \tau^{+} \bar{v}_{\tau}\right)=0.38-0.025 \mathrm{pb}$ were set for $m_{\mathrm{H}^{ \pm}}=80-160 \mathrm{GeV}$ and $m_{\mathrm{H}^{ \pm}}=180-600 \mathrm{GeV}$, as shown in Fig. 4 (left) and Fig. 4 (middle), respectively. By combining the $\mu \tau_{\mathrm{h}}, \ell+\mathrm{jets}$, and $\ell \ell^{\prime}$ final states and assuming $\mathscr{B}\left(\mathrm{H}^{+} \rightarrow \mathrm{t} \overline{\mathrm{b}}\right)=100 \%$, the first experimental result on the $\mathrm{H}^{+} \rightarrow \mathrm{t} \overline{\mathrm{b}}$ decay mode was derived, setting 95\% CL upper limits of $\sigma\left(\mathrm{pp} \rightarrow \overline{\mathrm{t}}(\mathrm{b}) \mathrm{H}^{+}\right)=$ $2.0-0.13 \mathrm{pb}$ for the mass range $m_{\mathrm{H}^{ \pm}}=180-600 \mathrm{GeV}$. These results, shown in Fig. 4 (right), were also interpreted in different MSSM benchmark scenarios and used to set exclusion limits in the $m_{\mathrm{H}^{ \pm}}-\tan \beta$ plane, while a lower bound on the charged Higgs boson mass of about $155 \mathrm{GeV}$ 

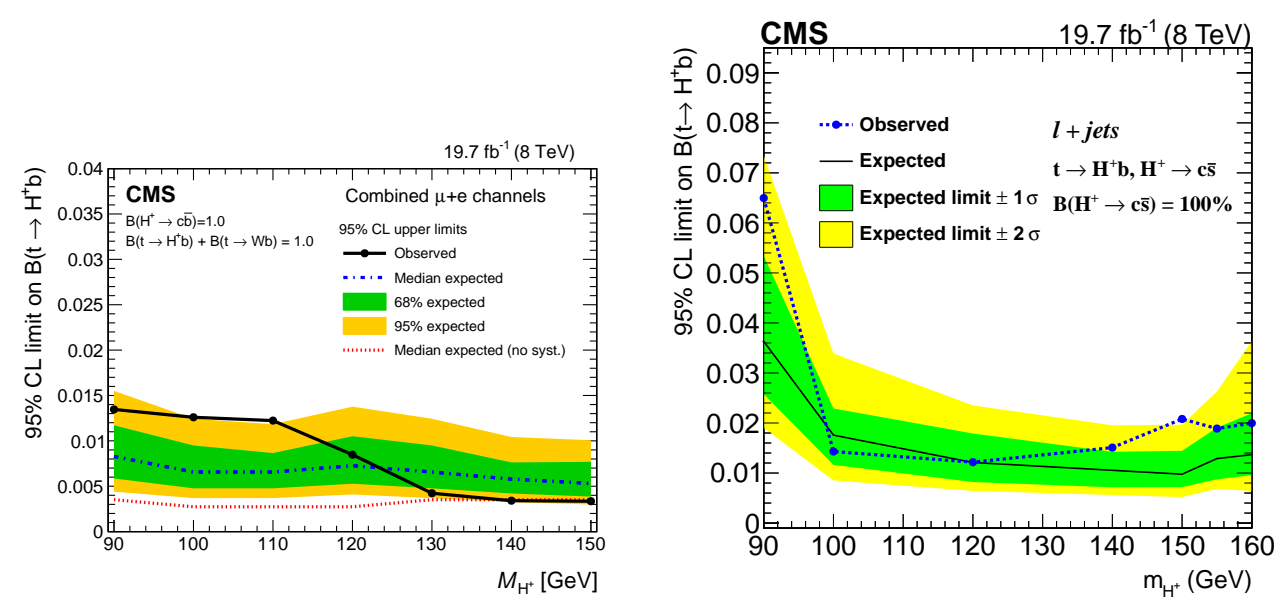

Figure 3: Exclusion limits on $\mathscr{B}\left(\mathrm{t} \rightarrow \mathrm{bH}^{+}\right)$as a function of $m_{\mathrm{H}^{+}}$assuming $\mathscr{B}\left(\mathrm{H}^{+} \rightarrow \mathrm{c} \overline{\mathrm{b}}\right)=100 \%$ (left) and $\mathscr{B}\left(\mathrm{H}^{+} \rightarrow \mathrm{c} \overline{\mathrm{s}}\right)=100 \%$ (right), using Run 1 legacy data. Taken from Refs. [30, 31].

was set under the assumption that $m_{\mathrm{h}}=125 \pm 3 \mathrm{GeV}$. Using the same assumption the lightstop scenario was also excluded for $m_{\mathrm{H}^{+}} \leq 160 \mathrm{GeV}$, while the low- $M_{H}$ scenario $[33,34]$ was completely excluded for $m_{\mathrm{H}^{0}}=125 \pm 3 \mathrm{GeV}$.
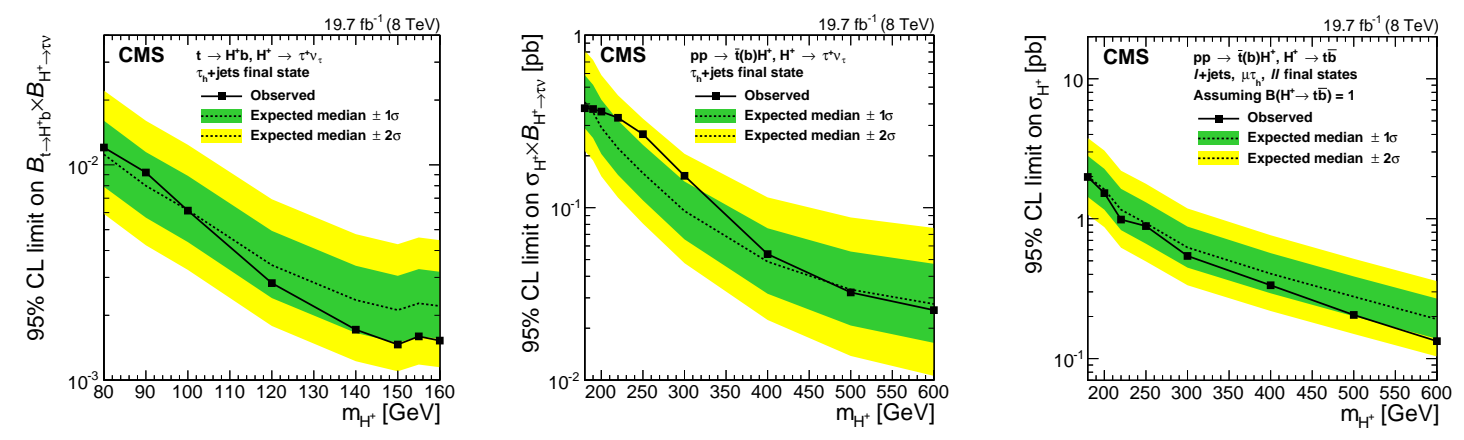

Figure 4: Model-independent upper limits at $95 \% \mathrm{CL}$ on $\mathscr{B}\left(\mathrm{t} \rightarrow \mathrm{bH}^{+}\right) \cdot \mathscr{B}\left(\mathrm{H}^{+} \rightarrow \tau^{+} \bar{v}_{\tau}\right)$ (left) and on $\sigma\left(\mathrm{pp} \rightarrow \overline{\mathrm{t}}(\mathrm{b}) \mathrm{H}^{+}\right) \cdot \mathscr{B}\left(\mathrm{H}^{+} \rightarrow \tau^{+} \bar{v}_{\tau}\right)$ (middle) as a function of $m_{\mathrm{H}^{+}}$for the $\mathrm{H}^{+} \rightarrow \tau^{+} \bar{v}_{\tau}$ search in the $\tau_{\mathrm{h}}+$ jets final state, using Run 1 legacy data. Upper limits at 95\% CL on $\sigma\left(\mathrm{pp} \rightarrow \overline{\mathrm{t}}(\mathrm{b}) \mathrm{H}^{+}\right)$ as a function of $m_{\mathrm{H}^{+}}$for the combination of the $\mu \tau_{\mathrm{h}}, \ell+\mathrm{jets}$, and $\ell \ell^{\prime}$ final states and assuming $\mathscr{B}\left(\mathrm{H}^{+} \rightarrow \mathrm{t} \overline{\mathrm{b}}\right)=100 \%$ (right). Taken from Ref. [32].

\section{Run 2 results}

On December 2018 the CMS experiment recorded the last collisions to bring to a close a very successful LHC second running period (Run 2) that began in 2015, with the LHC delivering to CMS more than $160 \mathrm{fb}^{-1}$ of proton-proton collisions at $\sqrt{s}=13 \mathrm{TeV}$. The first CMS Run 2 search for charged Higgs bosons in decays of $\mathrm{H}^{+} \rightarrow \tau^{+} \bar{v}_{\tau}$ focused on the fully hadronic final state and was performed using $12.9 \mathrm{fb}^{-1}$ of data collected in 2016 at $\sqrt{s}=13 \mathrm{TeV}$ [35]. Both light-mass and heavy-mass regions were explored by exploiting the fact that their experimental final states 
are similar. Events were selected in a final state containing at least three jets of which at least one was required to be b-tagged, an energetic $\tau_{\mathrm{h}}$ with one charged hadron (1-prong) and a polarisationmotivated discriminant, and large $p_{\mathrm{T}}^{\text {miss }}$. In addition, an angular discriminant relating the $\tau_{\mathrm{h}}, p_{\mathrm{T}}^{\text {miss }}$, and the three highest $p_{\mathrm{T}}$ jets in the event was employed motivated from possible mismeasurements of the jet momenta which may give rise to fake $p_{\mathrm{T}}^{\text {miss }}$. Events containing any isolated electrons or muons were rejected. The search strategy was to perform a binned ML fit to the transverse mass reconstructed from the $\tau_{\mathrm{h}}$ and $p_{\mathrm{T}}^{\text {miss }}$ objects. The resulting fit yielded model-independent limits on $\mathscr{B}\left(\mathrm{t} \rightarrow \mathrm{bH}{ }^{+}\right) \cdot \mathscr{B}\left(\mathrm{H}^{+} \rightarrow \tau^{+} \bar{v}_{\tau}\right)$ for $m_{\mathrm{H}^{ \pm}}=80-160 \mathrm{GeV}$, and on $\sigma\left(\mathrm{pp} \rightarrow \overline{\mathrm{t}}(\mathrm{b}) \mathrm{H}^{+}\right) \cdot \mathscr{B}\left(\mathrm{H}^{+} \rightarrow \tau^{+} \bar{v}_{\tau}\right)$ for $m_{\mathrm{H}^{ \pm}}=180-3000 \mathrm{GeV}$, as shown in Fig. 5. These results were also interpreted in MSSM benchmark scenarios and used to set new exclusion limits in the $m_{\mathrm{H}^{ \pm}}-\tan \beta$ plane.
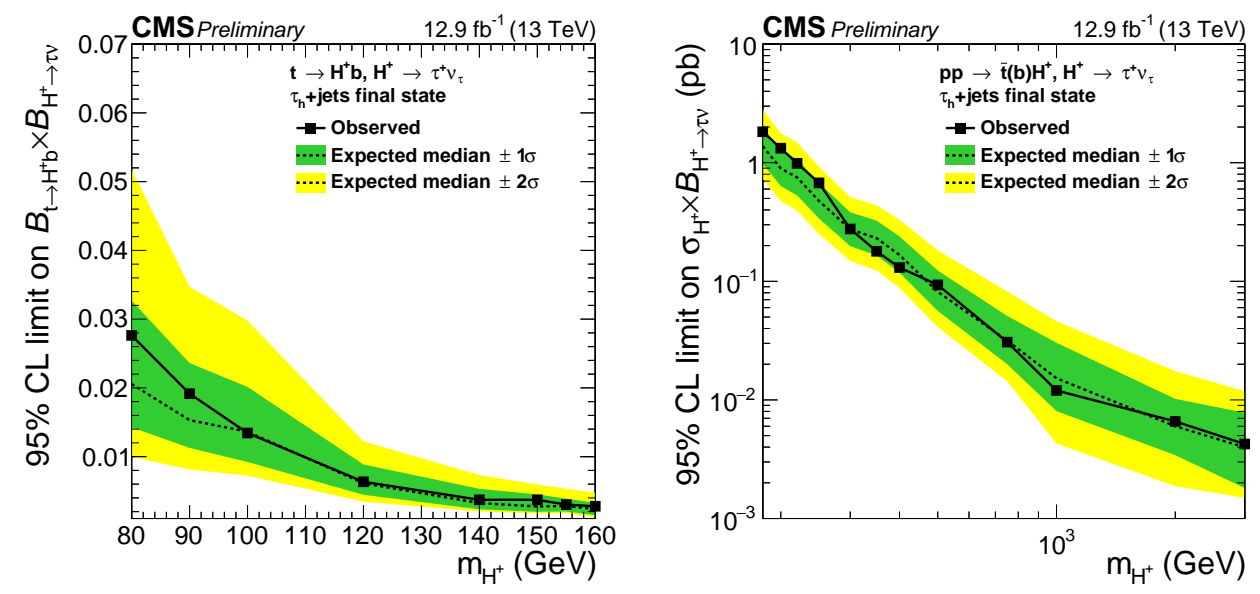

Figure 5: Model-independent upper limits at $95 \% \mathrm{CL}$ on $\mathscr{B}\left(\mathrm{t} \rightarrow \mathrm{bH}^{+}\right) \cdot \mathscr{B}\left(\mathrm{H}^{+} \rightarrow \tau^{+} \bar{v}_{\tau}\right)$ (left) and on $\sigma\left(\mathrm{pp} \rightarrow \overline{\mathrm{t}}(\mathrm{b}) \mathrm{H}^{+}\right) \cdot \mathscr{B}\left(\mathrm{H}^{+} \rightarrow \tau^{+} \bar{v}_{\tau}\right)$ (right) as a function of $m_{\mathrm{H}^{+}}$for the $\mathrm{H}^{+} \rightarrow \tau^{+} \bar{v}_{\tau}$ using Run 2 data collected in 2016. Taken from Ref. [35].

An updated search was recently performed with the $\mathrm{H}^{+} \rightarrow \tau^{+} \bar{v}_{\tau}$ decay mode and is presented here for the first time [36], based on data collected at $\sqrt{s}=13 \mathrm{TeV}$ in 2016 and corresponding to an integrated luminosity of $35.9 \mathrm{fb}^{-1}$. The search, which includes the first CMS results for the intermediate-mass region, was conducted in three orthogonal final states; a hadronic final state where events with leptons were vetoed $\left(\tau_{\mathrm{h}}+\right.$ jets $)$, a leptonic final state with a $\tau_{\mathrm{h}}$ where events with additional leptons were vetoed $\left(\ell+\tau_{\mathrm{h}}\right)$, and a leptonic final state without a $\tau_{\mathrm{h}}$ where events with a $\tau_{\mathrm{h}}$ or additional leptons were vetoed $\left(\ell+\right.$ no $\left.\tau_{\mathrm{h}}\right)$. The event selection and categorisation strategies were chosen separately for each final state to efficiently discriminate against the background events, while ensuring a sufficient signal selection efficiency.

For the $\tau_{\mathrm{h}}+$ jets final state, events were required to contain an energetic $\tau_{\mathrm{h}}$ with one charged hadron (1-prong), at least three hadronic jets with at least one of which being b-tagged, and large $p_{\mathrm{T}}^{\text {miss }}$. Any event with isolated electrons or muons passing loose identification and isolation criteria was rejected. Additionally, a selection relating $\Delta \phi\left(\tau_{\mathrm{h}}, p_{\mathrm{T}}^{\text {miss }}\right)$ and $\Delta \phi\left(\right.$ jet $\left._{n}, p_{\mathrm{T}}^{\text {miss }}\right)$ was applied, where the index $n$ runs over the three highest $p_{\mathrm{T}}$ jets $\left(\mathrm{jet}_{n}\right)$ in the event. This angular discriminant was used to suppress QCD multijet events, which typically contain a hadronic jet misidentified as a $\tau_{\mathrm{h}}$ and another hadronic jet recoiling in the opposite direction, thus giving rise to fake $p_{\mathrm{T}}^{\text {miss }}$. The 
selected events were classified into two categories, based on the value of the polarisation variable $R_{\tau}=p_{\mathrm{T}}^{\text {track }} / p_{\mathrm{T}}^{\tau_{\mathrm{h}}}$. This event categorisation was used to exploit the helicity correlations emerging from the opposite polarisation states of tau leptons originating from $\mathrm{W}$ and $\mathrm{H}^{ \pm}$decays [37].

For the $\ell+\tau_{\mathrm{h}}$ final state, events were required to contain electrons (muons) passing tight (medium) identification and tight isolation requirements. Events with any additional electrons or muons that passed loose identification and isolation criteria were vetoed. The presence of a $\tau_{\mathrm{h}}$ was required, along with large $p_{\mathrm{T}}^{\text {miss }}$, as well as one, two, or three hadronic jets with at least one of them b-tagged. The selected events were classified into several categories, with three categories defined based on the jet multiplicity and the number of jets passing the $b$ tagging requirement; one jet which is also identified as $a b$ jet $(1 j 1 b)$, at least two jets and one $b$ jet $(\geq 2 j 1 b)$, and at least 2 jets and at least $2 \mathrm{~b}$ jets $(\geq 2 \mathrm{j} \geq 2 \mathrm{~b})$. A second categorisation was performed in bins of $p_{\mathrm{T}}^{\text {miss }}$ (70-100, $100-150$, and $\geq 150 \mathrm{GeV}$ ), which together with the separate electron and muon final states give a total of 18 categories. This categorisation was found to improve significantly the search sensitivity, especially in the light-mass region, where efficient discrimination against backgrounds is essential.

For the $\ell+$ no $\tau_{\mathrm{h}}$ final state, events were selected using the same criteria as for the $\ell+\tau_{\mathrm{h}}$ final state, except for the following differences. Events with a $\tau_{\mathrm{h}}$ were vetoed and two or three jets were required, each well separated from the selected lepton. Higher jet multiplicities were rejected to accommodate other more sensitive searches performed by CMS, such as the $\mathrm{H}^{+} \rightarrow \mathrm{t} \overline{\mathrm{b}}$ decay mode. At least one of the jets was required to be b-tagged, while the presence of large $p_{\mathrm{T}}^{\text {miss }}$ was employed to greatly suppress QCD multijet events with jets misidentified as leptons. Additional angular selections were applied in the azimuthal plane, requiring large separation between the selected lepton and $p_{\mathrm{T}}^{\text {miss }}$ to suppress non-prompt muons from $\mathrm{b}$ hadron decays, while a large separation between the leading jet and $p_{\mathrm{T}}^{\text {miss }}$ was also used to efficiently discriminate against fake leptons from QCD multijet processes. Finally, events where the minimum separation between the selected lepton and any of the jets in the azimuthal plane were found to be in a back-to-back configuration were rejected, in order to suppress jet mismeasurement effects.

A simultaneous binned ML fit was performed over all the 36 categories in the three final states ( 2 from $\tau_{\mathrm{h}}+$ jets, 18 from $\ell+\tau_{\mathrm{h}}, 16$ from $\ell+$ no $\tau_{\mathrm{h}}$ ) using the transverse mass distribution from the $p_{\mathrm{T}}$ of the reconstructed $\tau_{\mathrm{h}}$ or isolated lepton $(\ell=\mathrm{e}, \mu)$ and its azimuthal angle with respect to $\vec{p}_{\mathrm{T}}^{\text {miss }}$. No significant excess was observed in any of the categories considered, and the result of the simultaneous fit was found to agree with the SM expectations. The combined 95\% CL modelindependent upper limits on $\sigma_{\mathrm{H}^{ \pm}} \cdot \mathscr{B}\left(\mathrm{H}^{ \pm} \rightarrow \tau^{ \pm} v_{\tau}\right)$ are shown in Fig. 6 (left), with the observed limit on $\mathscr{B}\left(\mathrm{t} \rightarrow \mathrm{bH} \mathrm{H}^{ \pm}\right) \cdot \mathscr{B}\left(\mathrm{H}^{ \pm} \rightarrow \tau^{ \pm} v_{\tau}\right)$ ranging from $5.97-0.0048 \mathrm{pb}$ for $m_{\mathrm{H}^{ \pm}}=80-3000 \mathrm{GeV}$. For the light-mass region $\left(m_{\mathrm{H}^{ \pm}}=80-160 \mathrm{GeV}\right)$ this corresponds to $\mathscr{B}\left(\mathrm{t} \rightarrow \mathrm{bH}^{+}\right) \cdot \mathscr{B}\left(\mathrm{H}^{+} \rightarrow\right.$ $\left.\tau^{+} \bar{v}_{\tau}\right)=0.36-0.08 \%$, making it the most stringent limit set by the CMS Collaboration to date. In the intermediate-mass region $\left(m_{\mathrm{H}^{ \pm}}=165-175 \mathrm{GeV}\right)$, which included for the first time in $\mathrm{H}^{ \pm}$ searches by CMS, the limit is $\mathscr{B}\left(\mathrm{t} \rightarrow \mathrm{bH} \mathrm{H}^{+}\right) \cdot \mathscr{B}\left(\mathrm{H}^{+} \rightarrow \tau^{+} \bar{v}_{\tau}\right)=1.01-0.52 \%$. The drop in the expected and observed limits is an experimental feature originating from the fact that LO signal samples were used here, instead of the next-to-leading order (NLO) samples used in the light-mass and heavy-mass regions. This dip is mitigated but not eradicated by the LO-to-NLO corrections extrapolated from the surrounding mass regions. In the heavy-mass region $\left(m_{\mathrm{H}^{ \pm}} \geq 180 \mathrm{GeV}\right)$ the limit corresponds to $\mathscr{B}\left(\mathrm{t} \rightarrow \mathrm{bH}^{+}\right) \cdot \mathscr{B}\left(\mathrm{H}^{+} \rightarrow \tau^{+} \bar{v}_{\tau}\right)=0.86-0.005 \%$. These results were interpreted in the MSSM $m_{\mathrm{h}}^{\text {mod- }}$ benchmark scenario [34] by comparing the observed limit on the 
$\mathrm{H}^{ \pm}$cross section to the theoretical cross sections predicted in this scenario [38 -42], and are shown in Fig. 6 (right).
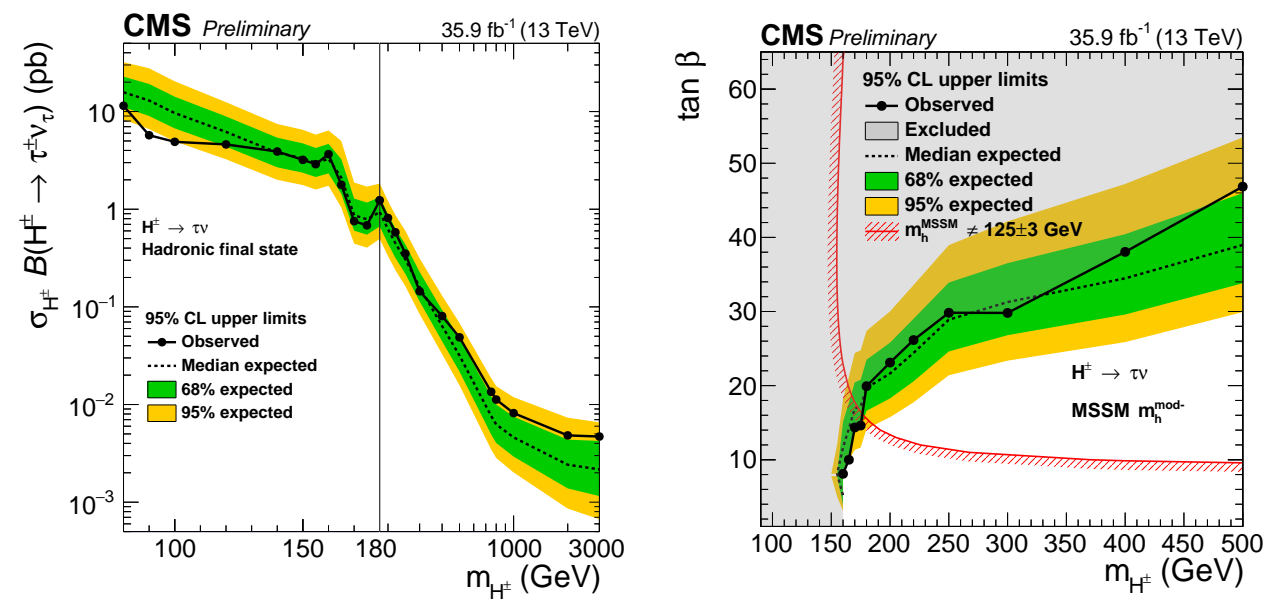

Figure 6: The 95\% CL exclusion limits on $\sigma_{\mathrm{H}^{ \pm}} \cdot \mathscr{B}\left(\mathrm{H}^{ \pm} \rightarrow \tau^{ \pm} v_{\tau}\right)$ as a function of $m_{\mathrm{H}^{+}}$for the $\mathrm{H}^{+} \rightarrow \tau^{+} \bar{v}_{\tau}$ decay mode using Run 2 data collected in 2016 (left). Their interpretation in the MSSM $m_{\mathrm{h}}^{\text {mod- }}$ benchmark scenario is also shown (right). The region below the red line is excluded Assuming under the assumption that the observed neutral Higgs boson is the light CP-even 2HDM Higgs boson with $m_{\mathrm{H}^{0}}=125 \pm 3 \mathrm{GeV}$. Taken from Ref. [36].

\section{References}

[1] ATLAS Collaboration, "Observation of a new particle in the search for the standard model Higgs boson with the ATLAS detector at the LHC", Phys. Lett. B $\mathbf{7 1 6}$ (2012) 1.

[2] CMS Collaboration, "Observation of a new boson at a mass of $125 \mathrm{GeV}$ with the CMS experiment at the LHC", Phys. Lett. B 716 (2012) 30.

[3] CMS Collaboration, "Observation of a new boson with mass near $125 \mathrm{GeV}$ in pp collisions at $\sqrt{s}=7$ and 8 TeV", JHEP 06 (2013) 081.

[4] P. W. Higgs, "Broken symmetries, massless particles and gauge fields", Phys. Lett. 12132.

[5] P. W. Higgs, "Broken symmetries and the masses of gauge bosons", Phys. Rev. Lett. 13508.

[6] G. S. Guralnik, C. R. Hagen, and T. W. B. Kibble, "Global conservation laws and massless particles", Phys. Rev. Lett. 13585.

[7] P. W. Higgs, "Spontaneous symmetry breakdown without massless bosons", Phys. Rev. 1451156.

[8] T. W. B. Kibble, "Symmetry breaking in non-Abelian gauge theories", Phys. Rev. 1551554.

[9] F. Englert and R. Brout, "Broken symmetry and the mass of gauge vector mesons", Phys. Rev. Lett. 13321.

[10] CMS Collaboration, "Constraints on the spin-parity and anomalous HVV couplings of the Higgs boson in proton collisions at 7 and 8 TeV", Phys. Rev. D 92 (2015) 012004. 
[11] ATLAS and CMS Collaborations, "Combined measurement of the Higgs boson mass in pp collisions at $\sqrt{s}=7$ and $8 \mathrm{TeV}$ with the ATLAS and CMS experiments", Phys. Rev. Lett. 114 (2015) 191803.

[12] ATLAS Collaboration, "Study of the spin and parity of the Higgs boson in diboson decays with the ATLAS detector", Eur. Phys. J. C 75 (2015) 476.

[13] ATLAS and CMS Collaborations, "Measurements of the Higgs boson production and decay rates and constraints on its couplings from a combined ATLAS and CMS analysis of the LHC pp collision data at $\sqrt{s}=7$ and $8 \mathrm{TeV}$ ", JHEP $\mathbf{0 8}$ (2016) 045.

[14] CMS Collaboration, "Measurements of properties of the Higgs boson decaying into the four-lepton final state in pp collisions at $\sqrt{s}=13 \mathrm{TeV} "$, JHEP 11 (2017) 047.

[15] V. D. Barger, J. L. Hewett, and R. J. N. Phillips, "New Constraints on the Charged Higgs Sector in Two Higgs Doublet Models”, Phys. Rev. D41 (1990) 3421-3441.

[16] A. Djouadi, "The anatomy of electro-weak symmetry breaking. II. the Higgs bosons in the minimal supersymmetric model", Phys. Rept. 459 (2008) 1.

[17] G. Senjanovic and R. N. Mohapatra, "Exact Left-Right Symmetry and Spontaneous Violation of Parity", Phys. Rev. D 12 (1975) 1502.

[18] J. F. Gunion, R. Vega, and J. Wudka, "Higgs triplets in the standard model", Phys. Rev. D 42 (1990) 1673.

[19] H. Georgi and M. Machacek, "Doubly charged Higgs bosons", Nucl. Phys. B 262 (1985) 463.

[20] M. Aoki, S. Kanemura, K. Tsumura, and K. Yagyu, "Models of Yukawa interaction in the two Higgs doublet model, and their collider phenomenology", Phys. Rev. D80 (2009) 015017.

[21] C. Degrande et al., "Accurate predictions for charged Higgs production: Closing the $m_{\mathrm{h}^{ \pm}} \sim m_{\mathrm{t}}$ window”, Phys. Lett. B 772 (2017) 87.

[22] CMS Collaboration, "The CMS experiment at the CERN LHC", JINST 3 S08004.

[23] CMS Collaboration, "Particle-flow reconstruction and global event description with the CMS detector", JINST 12 (2017) P10003.

[24] M. Cacciari, G. P. Salam, and G. Soyez, “The anti- $k_{\mathrm{T}}$ jet clustering algorithm”, JHEP 04 (2008) 063.

[25] M. Cacciari, G. P. Salam, and G. Soyez, "FastJet user manual", Eur. Phys. J. C 721896.

[26] CMS Collaboration, "Reconstruction and identification of $\tau$ lepton decays to hadrons and $v_{\tau}$ at CMS”, JINST 11 (2016) P01019.

[27] CMS Collaboration, "Performance of reconstruction and identification of $\tau$ leptons decaying to hadrons and $v_{\tau}$ in pp collisions at $\sqrt{s}=13 \mathrm{TeV}$ ", JINST 13 (2018) P10005.

[28] CMS Collaboration, "Identification of heavy-flavour jets with the CMS detector in pp collisions at 13 TeV”, JINST 13 (2018) P05011.

[29] CMS Collaboration, "Performance of missing transverse momentum in pp collisions at $\sqrt{s}=13$ tev using the cms detector", CMS PAS JME-17-001 (2018).

[30] CMS Collaboration, "Search for a charged Higgs boson decaying to charm and bottom quarks in proton-proton collisions at $\sqrt{s}=8 \mathrm{TeV}$ ", JHEP 11 (2018) 115.

[31] CMS Collaboration, "Search for a light charged Higgs boson decaying to cs in pp collisions at $\sqrt{s}=8$ TeV”, JHEP 12 (2015) 178. 
[32] CMS Collaboration, "Search for a charged Higgs boson in pp collisions at $\sqrt{s}=8 \mathrm{TeV}$ ", JHEP 11 (2015) 018.

[33] LHC Higgs Cross Section Working Group Collaboration, "Handbook of LHC Higgs Cross Sections: 3. Higgs Properties",

[34] M. Carena et al., "MSSM Higgs Boson Searches at the LHC: Benchmark Scenarios after the Discovery of a Higgs-like Particle”, Eur. Phys. J. C73 (2013), no. 9, 2552.

[35] CMS Collaboration, "Search for charged higgs bosons with the $\mathrm{h}^{ \pm} \rightarrow \tau^{ \pm} v_{\tau}$ decay channel in the fully hadronic final state at $\sqrt{s}=13$ tev", CMS PAS HIG-16-031 (2016).

[36] CMS Collaboration, "Search for charged higgs bosons with the $\mathrm{h}^{ \pm} \rightarrow \tau^{ \pm} v_{\tau}$ decay channel in proton-proton collisions at $\sqrt{s}=13$ tev", CMS PAS HIG-18-014 (2018).

[37] D. P. Roy, “The hadronic tau decay signature of a heavy charged Higgs boson at LHC”, Phys. Lett. B 459 (1999) 607, arXiv: hep-ph/9905542.

[38] LHC Higgs Cross Section Working Group Collaboration, "Handbook of LHC Higgs cross sections: 4. deciphering the nature of the Higgs sector”, (2016). arXiv:1610.07922.

[39] M. Flechl et al., "Improved cross-section predictions for heavy charged Higgs boson production at the LHC”, Phys. Rev. D 91 (2015) 075015.

[40] C. Degrande, M. Ubiali, M. Wiesemann, and M. Zaro, "Heavy charged Higgs boson production at the LHC", JHEP 10 (2015) 145.

[41] S. Dittmaier, M. Kramer, M. Spira, and M. Walser, "Charged-Higgs-boson production at the LHC: NLO supersymmetric QCD corrections”, Phys. Rev. D 83 (2011) 055005.

[42] E. L. Berger, T. Han, J. Jiang, and T. Plehn, "Associated production of a top quark and a charged Higgs boson”, Phys. Rev. D 71 (2005) 115012. 